\title{
Representations of Intervals and Optimal Error Bounds*
}

\begin{abstract}
By L. B. Rall
Abstract. Classical methods of numerical analysis lead to approximate values and error bounds for computed results, while interval analysis provides lower and upper bounds for the exact values. Equivalence of these approaches is investigated on the basis of a general definition of an error function, and a way is given to find optimal approximation points $y^{*}$ in an interval $I$ and the corresponding minimum error bound $\varepsilon^{*}$. Conversely, it is shown that $l^{*}, \varepsilon^{*}$ can be used as alternative coordinates for the representation of the interval $I$, and the rules for interval arithmetic can be formulated in the resulting system. In particular, the arithmetic, harmonic, and geometric means of the endpoints of an interval turn out to be the optimal approximation points corresponding to absolute error, relative error, and relative precision, respectively. The rules for interval arithmetic are given explicitly in the resulting coordinate systems. It is also shown that calculations performed in interval arithmetic generally lead to smaller intervals than predicted by certain a priori bounds.
\end{abstract}

1. Intervals and Error Bounds. Since real transformations $y=f(x)$ cannot be evaluated exactly in general, a central problem of numerical analysis is to find reliable approximations $y^{*}$ to $y$. In classical numerical analysis, the reliability of $y^{*}$ is determined on the basis of an error bound $\varepsilon^{*}$ relative to some measure of error, a concept which will be formulated precisely below. The more recently developed methods of interval analysis [2], [3], approach this problem in the following way: It is assumed that $x \in J$, a possibly degenerate interval, and one computes $I=F(J)$ using an interval operator $F$ which includes $f$ on $J$ in the sense that

$$
f(J)=\{y \mid y=f(x), x \in J\} \subset F(J) .
$$

Thus, $y \in I$ for the exact value $y=f(x)$, so that, for

$$
a=i(I)=\inf \{x \mid x \in I\}, \quad b=s(I)=\sup \{x \mid x \in I\},
$$

one has

$$
a \leqslant y \leqslant b,
$$

which gives lower and upper bounds for the value sought.

The equivalence of the results of these approaches is evident: Given (1.3), it is usually easy to compute an approximate value $y^{*}$ and an upper bound $\varepsilon^{*}$ for its possible error; examples will be given below. Similarly, given $y^{*}, \varepsilon^{*}$, then it is

Received March 26, 1982.

1980 Mathematics Subject Classification. Primary 65G10, 41A50.

Key words and phrases. Optimal approximations, optimal error bounds, interval arithmetic, absolute error, relative error, percentage error, relative precision, elementary means, excess width.

*Research sponsored in part by the U. S. Army under Contract No. DAAG29-80-C-0041 and Danish National Science Research Council Grant No. 511-15849. 
conversely possible to assert that the exact value $y$ lies in some interval $I$. Calculations in interval analysis are based ultimately (but not completely) on interval arithmetic [2], [3], which is usually expressed in the standard format $I=[a, b]$, $J=[c, d]$ for intervals according to (1.2). The rules are:

(i) Addition

$$
I+J=[a, b]+[c, d]=[a+b, c+d] .
$$

(ii) Subtraction

$$
I-J=[a, b]-[c, d]=[a-d, b-c] .
$$

(iii) Multiplication

(1.6) $I \cdot J=[a, b] \cdot[c, d]=[\min \{a c, a d, b c, b d\}, \max \{a c, a d, b c, b d\}]$.

(iv) Reciprocation for intervals $J$ such that $0 \notin J$,

$$
J^{-1}=\left[d^{-1}, c^{-1}\right] \text { if } c d>0 .
$$

Division is defined by the compound operation $I / J=I \cdot J^{-1}$ for $0 \notin J$. These operations could just as well be defined in terms of the functionals $i, s$ introduced in (1.2); for example, (1.5) can be written

$$
i(I-J)=i(I)-s(J), \quad s(I-J)=s(I)-i(J) .
$$

It will be shown below that the pair $y^{*}, \varepsilon^{*}$ arising from various classical error estimates can be used as an alternate coordinate system for the representation of intervals, and the rules of interval arithmetic can be formulated in terms of these coordinates, which leads to simple rules for certain operations.

2. Error Functions and Optimal Error Bounds. Measurement of error of approximate values will be made according to functions satisfying the following definition.

Definition 2.1. A continuous function $E(y, z)$ is a measure of the error of the approximation of the real number $z$ by a real number $y$ if

(i) $E(y, z) \geqslant 0$,

(ii) $E(z, z)=0$, and

(iii) $E(y, z)$ is a strictly monotone increasing function of $|y-z|$ for fixed $z$. Under these conditions, $E(x, z)$ will be called an error function.

Given an error function $E$ and the knowledge that the point $z$ belongs to an interval $I$, the concepts of optimal approximation and error bound can be developed.

Definition 2.2. The error of approximation of points $z \in I$ by a real number $y$ is defined to be

$$
\varepsilon(y)=\max \{E(y, z) \mid z \in I\} .
$$

Definition 2.3. A point $y^{*} \in I$ is said to be optimal in $I$ for the error function $E$ if

$$
\varepsilon\left(y^{*}\right)=\min \{\varepsilon(y) \mid y \in I\}=\min \{\max \{E(y, z) \mid z \in I\} \mid y \in I\} .
$$

THEOREM 2.1. The optimal point $y^{*} \in I$ exists and is the unique solution $y=y^{*}$ in $I=[a, b]$ of the equation

$$
E(y, a)=E(y, b) .
$$

Proof. If $a=b$, then $y^{*}=a=b$; otherwise, the function

$$
h(y)=E(y, a)-E(y, b)
$$


is continuous, and $h(a)<0, h(b)>0$ by hypothesis. Thus, $h\left(y^{*}\right)=0$ for some $y^{*} \in I$, and $y=y^{*}$ consequently satisfies (2.3). Now, suppose that $y<y^{*}$. It follows that

$$
E(y, b)>E\left(y^{*}, b\right)=E\left(y^{*}, a\right),
$$

since $E$ is strictly monotone increasing in $|y-b|$, and if $y>y^{*}$, then similarly

$$
E(y, a)>E\left(y^{*}, a\right)=E\left(y^{*}, b\right),
$$

from which the optimality and uniqueness of $y^{*}$ follow. Q.E.D.

Definition 2.4. The value

$$
\varepsilon^{*}=\varepsilon\left(y^{*}\right)=E\left(y^{*}, a\right)=E\left(y^{*}, b\right)
$$

obtained for optimal $y^{*} \in I$ is called the optimal error bound on $I$ for the error function $E$. It is the minimum of the maximum possible error on $I$.

Thus, finding the optimal approximation $y^{*}$ to points of $I$ and the corresponding minimum error bound $\varepsilon^{*}$ simply requires the solution of the equation (2.3) and the subsequent evaluation of (2.7). In order to introduce given $y^{*}, \varepsilon^{*}$ as alternative coordinates for the representation of $I$, it is sufficient to note that the inverse functions

$$
\begin{aligned}
l(\varepsilon) & =\left\{y \mid E\left(y^{*}, y\right)=\varepsilon, y \leqslant y^{*}\right\}, & & \varepsilon \geqslant 0, \\
u(\varepsilon) & =\left\{y \mid E\left(y^{*}, y\right)=\varepsilon, y \geqslant y^{*}\right\}, & & \varepsilon \geqslant 0,
\end{aligned}
$$

are single-valued and continuous by the hypotheses on the error function $E$, so that $y^{*}, \varepsilon^{*}$ are optimal for the interval $I$ for which

$$
a=i(I)=l\left(\varepsilon^{*}\right), \quad b=s(I)=u\left(\varepsilon^{*}\right),
$$

and thus $I=[a, b]=\left[l\left(\varepsilon^{*}\right), u\left(\varepsilon^{*}\right)\right]$ in standard format, which provides the transformations from $y^{*}=y^{*}(I), \varepsilon^{*}=\varepsilon^{*}(I)$ to $a=i(I), b=s(I)$ coordinates.

3. Absolute Error. The simplest error function is the absolute error

$$
E(y, z)=|y-z|
$$

of the approximation of $z$ by $y$. As is well known, the results of Section 2 lead to the optimal point and error bound

$$
y^{*}=m(I)=(a+b) / 2, \quad \varepsilon^{*}=\alpha(I)=(b-a) / 2
$$

on $I=[a, b]$, where $m=m(I)$ is the midpoint of $I$, and $\alpha=\alpha(I)$ is the halfwidth of $I$, the width of $I$ being $w(I)=b-a$ [2], [3]. The transformation from $m, \alpha$ coordinates to standard format is given by

$$
a=i(I)=m-\alpha, \quad b=s(I)=m+\alpha .
$$

In this coordinate system, intervals are customarily denoted by

$$
I=m(I) \pm \alpha(I) \text {. }
$$

The rules for interval arithmetic are easily expressed in $m, \alpha$ coordinates.

(i) Addition

$$
m(I+J)=m(I)+m(J), \quad \alpha(I+J)=\alpha(I)+\alpha(J) .
$$

(ii) Subtraction

$$
m(I-J)=m(I)-m(J), \quad \alpha(I-J)=\alpha(I)+\alpha(J) .
$$


(iii) Multiplication: From

$$
\begin{array}{ll}
m_{1}(I \cdot J)=m(I) m(J)-\alpha(I) m(J), & \alpha_{1}=|m(I) \alpha(J)-\alpha(I) \alpha(J)|, \\
m_{2}(I \cdot J)=m(I) m(J)-m(I) \alpha(J), & \alpha_{2}=|\alpha(I) m(J)-\alpha(I) \alpha(J)|, \\
m_{3}(I \cdot J)=m(I) m(J)+\alpha(I) \alpha(J), & \alpha_{3}=|\alpha(I) m(J)+m(I) \alpha(J)|, \\
m_{4}(I \cdot J)=m(I) m(J)-\alpha(I) \alpha(J), & \alpha_{4}=|\alpha(I) m(J)-m(I) \alpha(J)|, \\
m_{5}(I \cdot J)=m(I) m(J)+m(I) \alpha(J), & \alpha_{5}=|\alpha(I) m(J)+\alpha(I) \alpha(J)|, \\
m_{6}(I \cdot J)=m(I) m(J)+\alpha(I) m(J), & \alpha_{6}=|m(I) \alpha(J)+\alpha(I) \alpha(J)|,
\end{array}
$$

pick a pair $m_{i}, \alpha_{i}$ for which

$$
\alpha_{i}=\max \left\{\alpha_{1}, \alpha_{2}, \alpha_{3}, \alpha_{4}, \alpha_{5}, \alpha_{6}\right\} .
$$

(iv) Reciprocation: For $0 \notin J, m(J)^{2}-\alpha(J)^{2}>0$,

$$
\begin{aligned}
m\left(J^{-1}\right) & =m(J) /\left(m(J)^{2}-\alpha(J)^{2}\right), \\
\alpha\left(J^{-1}\right) & =\alpha(J) /\left(m(J)^{2}-\alpha(J)^{2}\right), \quad \text { if } m(J)^{2}>\alpha(J)^{2} .
\end{aligned}
$$

Remark 3.1. In $m, \alpha$ coordinates, addition and subtraction are one-component operations: $m(I \pm J)$ depends only on $m(I), m(J)$, and $\alpha(I \pm J)$ similarly is a function of $\alpha(I), \alpha(J)$ only. Simplifications of this type will be seen below for multiplication and reciprocation. However, Ratschek [5] has proved that there are no coordinate systems for intervals in which both addition and multiplication are one-component operations.

Remark 3.2. The rule for multiplication in midpoint-halfwidth coordinates looks complicated. However, it is comparable to (1.6), which requires the selection of a maximum and minimum value from four elements, while here only finding the largest of six nonnegative numbers is required. Furthermore, since $\alpha(I), \alpha(J) \geqslant 0$, an analysis of the signs of $m(I), m(J)$ will eliminate some of the $\alpha_{j}$ at once.

The midpoint $m(I)$ of $I$, at which the maximum bound for the absolute error is a minimum, is, of course, the arithmetic mean (average) of the endpoints $a, b$ of $I$.

4. Relative and Percentage Error. For positive intervals $(a=i(I)>0)$, a useful measure of error in many applications is the relative error of $y$ as an approximation to $z$, for which the corresponding error function is

$$
E(y, z)=\left|\frac{y-z}{z}\right| \text {. }
$$

Note that this error function is asymmetric; in general, $E(y, z) \neq E(z, y)$. Another commonly employed error function is the percentage error

$$
P(y, z)=100 E(y, z)=100\left|\frac{y-z}{z}\right| .
$$

For each of these error functions, Eq. (2.3) gives

$$
y^{*}=h(y)=\frac{2 a b}{a+b},
$$

the harmonic point of the interval $I=[a, b]$ as the optimal point, and the optimal error bounds

$$
\varepsilon^{*}=r(I)=(b-a) /(a+b), \quad \varepsilon^{*}=p(I)=100 r(I),
$$


corresponding to (4.1) and (4.2), respectively. The quantity $r(I)=(b-a) /(a+b)$ will be called the relative width of the interval $I=[a, b]$. The transformations from $h, r$ coordinates back to the standard format are given by

$$
a=i(I)=h(I) /(1+r(I)), \quad b=s(I)=h(I) /(1-r(I)) \text {. }
$$

Similar formulas can be obtained for the $h, p$ coordinates simply by setting $r(I)=p(I) / 100$ in (4.5). In these systems, one can write the interval $I$ in the forms

$$
I=h(I) \text { r.e. } r(I), \quad I=h(I) \pm p(I) \%,
$$

respectively. Because of the simple relationship between $p(I)$ and $r(I)$, the rules for interval arithmetic will be given for the $h, r$ coordinates only.

(i) Addition

$$
\begin{aligned}
& h(I+J)=\frac{(h(I)+h(J))^{2}-(h(I) r(J)+h(J) r(I))^{2}}{h(I)+h(J)-\left(h(I) r(J)^{2}+h(J) r(I)^{2}\right)}, \\
& r(I+J)=\frac{h(I) r(I)+h(J) r(J)-\left(h(I) r(I) r(J)^{2}+h(J) r(J) r(I)^{2}\right)}{h(I)+h(J)-\left(h(I) r(J)^{2}+h(J) r(I)^{2}\right)} .
\end{aligned}
$$

(ii) Subtraction is defined only for $I-J$ positive,

$$
\begin{aligned}
& h(I-J)=\frac{(h(I)-h(J))^{2}-(h(I) r(J)+h(J) r(I))^{2}}{h(I)-h(J)-\left(h(I) r(J)^{2}+h(J) r(I)^{2}\right)}, \\
& r(I-J)=\frac{h(I) r(I)+h(J) r(J)-\left(h(I) r(I) r(J)^{2}+h(J) r(J) r(I)^{2}\right)}{h(I)-h(J)-\left(h(I) r(J)^{2}+h(J) r(I)^{2}\right)},
\end{aligned}
$$

provided $h(I)>h(J)+h(I) r(J)^{2}+h(J) r(I)^{2}$.

(iii) Multiplication

$$
h(I \cdot J)=\frac{h(I) h(J)}{1+r(I) r(J)}, \quad r(I \cdot J)=\frac{r(I)+r(J)}{1+r(I) r(J)} .
$$

(iv) Reciprocation

$$
h\left(J^{-1}\right)=\frac{1-r(J)^{2}}{h(J)}, \quad r\left(J^{-1}\right)=r(J) .
$$

The formulas (4.7) and (4.8) for addition and subtraction are too unwieldy to be of practical importance; however (4.9) and (4.10) are simple and direct, and multiplication and hence division require no sorting. Of course, for positive intervals in the standard format, $[a, b] \cdot[c, d]=[a c, b d]$ is also obtained directly.

The optimal point $h(I)$ here is the harmonic mean of the endpoints $a, b$ of $I$. The restriction to positive intervals is just to make sure that $0 \notin I$; analogous formulas hold for negative intervals with $b=s(I)<0$.

5. Relative Precision and Approximate Relative Precision. Olver [4] defines $y^{*}$ to be an approximation to $y$ with relative precision $\sigma$,

$$
y \cong y^{*} \text { r.p. } \sigma, \quad \sigma \geqslant 0,
$$

to mean that

$$
y^{*} e^{-\sigma} \leqslant y \leqslant y^{*} e^{\sigma}
$$


Similarly, approximate relative precision $\tau$,

$$
y \cong y^{*} \text { a.r.p. } \tau, \quad 0 \leqslant \tau<1,
$$

for the approximation of $y$ by $y^{*}$ means

$$
(1-\tau) y^{*} \leqslant y \leqslant y^{*} /(1-\tau)
$$

an interval relationship similar to (5.2). These recently introduced concepts lead to simple derivations of a priori error bounds for a number of important computational procedures [4]. The formulaton of the error functions corresponding to (5.1) and (5.3), and the derivation of optimal points and error bounds, can be made on the basis of the function

$$
M(y, z)=\max \left\{\frac{y}{z}, \frac{z}{y}\right\}, \quad y, z>0 .
$$

Here again, only positive intervals will be considered. The results apply to negative intervals as well, with the obvious modifications. Examination of the function $M(y, z)$ leads at once to the following conclusions.

LEMMA 5.1. For $y>0$ and fixed $z>0, M(y, z)$ is a monotone increasing function of $|y-z|$.

Proof. This follows at once from the representation

$$
M(y, z)=\frac{y^{2}+z^{2}+\left|y^{2}-z^{2}\right|}{2 y z}
$$

for $y, z>0$. Q.E.D.

The next proposition results from the same reasoning as in the proof of Theorem 2.1 .

LEMMA 5.2. On the positive interval $I=[a, b]$, the minimum $\rho(I)$ of the maximum value of $M(y, z)$ is attained for $y=y^{*}$ satisfying

$$
\frac{y}{a}=\frac{b}{y}
$$

that is,

$$
y^{*}=g(I)=\sqrt{a b}, \quad \rho(I)=\sqrt{b / a} .
$$

Definition 5.1. The point $g(I)=\sqrt{a b}$ is called the geometric point of a positive interval $I$, and $\rho(I)=\sqrt{b / a}$ is the ratio of $I$.

The following theorem is an immediate consequence of Definitions 2.1-2.4 and Lemmas 5.1-5.2.

THEOREM 5.1. If $f$ is a strictly monotone increasing function of $x$ for $x \geqslant 1$ such that $f(1)=0$, then

$$
E(y, z)=f(M(y, z))
$$

is an error function for $y, z>0$ with optimal point and error bound

$$
y^{*}=g(I), \quad \varepsilon^{*}=f(\rho(I))
$$

on each positive interval $I$. 
The conclusion of Theorem 5.1 applies immediately to relative precision, since (5.2) is equivalent to the error function

$$
E(y, z)=\ln (M(y, z)),
$$

for which the optimal error bound is

$$
\varepsilon^{*}=\sigma(I)=\ln (\rho(I)) .
$$

Similarly, from (5.4), the error function corresponding to approximate relative precision is

$$
E(y, z)=(M(y, z)-1) / M(y, z),
$$

which has the optimal value

$$
\varepsilon^{*}=\tau(I)=(\rho(I)-1) / \rho(I)
$$

on the positive interval $I$, again at the geometric point $y^{*}=g(I)$.

Thus,

$$
\rho(I)=e^{\sigma(I)}=1 /(1-\tau(I)),
$$

so the rules for interval arithmetic in $g(I), \sigma(I)$ or $g(I), \tau(I)$ coordinates come easily from the ones given below for the geometric point-ratio coordinates $g=g(I)$, $\rho=\rho(I)$. These lead to the representation

$$
I=g(I) * \rho(I),
$$

for positive intervals ( $*$ is a combination of $\div$ and $\times$ ), and the inverse transformations

$$
a=i(I)=g(I) / \rho(I), \quad b=s(I)=g(I) \rho(I) .
$$

The rules for interval arithmetic in $g, \rho$ coordinates are:

(i) Addition

$$
\begin{aligned}
& g(I+J)^{2}=(g(I)+g(J))^{2}-\frac{g(I) g(J)}{\rho(I) \rho(J)}(\rho(I)-\rho(J))^{2}, \\
& \rho(I+J)^{2}=\rho(I) \rho(J) \frac{g(I) \rho(I)+g(J) \rho(J)}{g(I) \rho(J)+g(J) \rho(I)} .
\end{aligned}
$$

(ii) Subtraction is defined only for $I-J$ positive,

$$
\begin{aligned}
g(I-J)^{2} & =(g(I)-g(J))^{2}-\frac{g(I) g(J)}{\rho(I) \rho(J)}(\rho(I) \rho(J)-1)^{2} \\
\rho(I-J)^{2} & =\frac{\rho(I)}{\rho(J)}\left(\frac{g(I) \rho(I) \rho(J)-g(J)}{g(I)-g(J) \rho(I) \rho(J)}\right) \\
& \text { if } g(I)-g(J) \rho(I) \rho(J)>0 .
\end{aligned}
$$

(iii) Multiplication

$$
g(I \cdot J)=g(I) g(J), \quad \rho(I \cdot J)=\rho(I) \rho(J) .
$$

(iv) Reciprocation

$$
g\left(J^{-1}\right)=1 / g(J)=g(J)^{-1}, \quad \rho\left(J^{-1}\right)=\rho(J) .
$$

Remark 5.1. In $g, \rho$ coordinates, multiplication and reciprocation (and hence division) are one-component operations. 
Remark 5.2. In the special case that $\rho(I)=\rho(J)$, formulas (5.18) for addition become

$$
g(I+J)=g(I)+g(J), \quad \rho(I+J)=\rho(I)=\rho(J) .
$$

Thus, addition is a single component operation in this case; furthermore, the second component is constant, as in reciprocation (5.21). As noted by Olver [4], in addition of numbers $A_{1}, A_{2}, \ldots, A_{n}$, the maximum relative or approximate relative precision can be assigned to all and the result by (5.22). Furthermore, in evaluating the convergent of a continued fraction of the form

$$
C_{n}=\frac{1}{A_{1}}+\frac{1}{A_{2}}+\cdots+\frac{1}{A_{n}},
$$

the maximum relative or approximate relative precision of $A_{1}, A_{2}, \ldots, A_{n}$ can be assigned to the result by (5.21) and (5.22).

Remark 5.3. The point $g(I)=\sqrt{a b}$ is, of course, the geometric mean of the endpoints of the positive interval $I=[a, b]$. It is interesting that the elementary mean values $m(I), h(I), g(I)$ turn out to be optimal points for the absolute error, relative (or percentage) error, and relative (or approximate relative) precision, respectively, all of which are useful measures of error in various applications. Furthermore, the fundamental inequality between these means [1] gives

$$
h(I) \leqslant g(I) \leqslant m(I),
$$

so that relative precision is a measure of error intermediate to relative and absolute error in a certain sense.

Remark 5.4. The $g, \rho$ coordinates for intervals are related to the midpoint-halfwidth coordinates logarithmically. For a positive interval $I=[a, b]$, define $\ln (I)=$ $[\ln (a), \ln (b)]$. Then,

$$
\ln (g(I))=m(\ln (I)), \quad \ln (\rho(I))=\alpha(\ln (I)) .
$$

There are a number of other relationships between the coordinate systems considered in this paper, for example,

$$
m\left(J^{-1}\right)=1 / h(J)=h(J)^{-1}, \quad \alpha\left(J^{-1}\right)=\alpha(J) / g(J)^{2} .
$$

In performing interval arithmetic, one should keep in mind the possibility of economy or convenience in using one coordinate system or another for the type of calculations actually being done, and the possibility of transformations between systems.

6. Excess Width of A Priori Bounds. Suppose that $I$ is a subinterval of $J$. The quantity

$$
e(J, I)=w(J)-w(I), \quad I \subset J,
$$

is called the excess width of $J$ over $I$. If $I$ is the smallest interval which contains the actual results for given input data, then one of course desires to produce an interval $J$ as the result of computation which has excess width over $I$ which is as small as possible. In some cases, intervals $J$ obtained by a priori error estimates will be wider than those computed by interval arithmetic. For example, Olver [4] defines absolute precision to mean the same as absolute error as considered in Section 3, that is

$$
a \cong \tilde{a} \text { a.p. } \alpha \Leftrightarrow a \in \tilde{a} \pm \alpha .
$$


If $b \cong \tilde{b}$ a.p. $\beta$, then Olver gives the formula

$$
a b \cong \tilde{a} \tilde{b} \text { a.p. }|\tilde{a}| \beta+|\tilde{b}| \alpha+\alpha \beta,
$$

which, in interval notation, defines the interval $K$ with

$$
\begin{aligned}
m(K) & =m(I) m(J), \\
\alpha(K) & =|m(I)| \alpha(J)+|m(J)| \alpha(I)+\alpha(I) \alpha(J) .
\end{aligned}
$$

For positive intervals $I, J$, formulas (3.7)-(3.8) give the simple results

$$
\begin{aligned}
m(I \cdot J) & =m(I) m(J)+\alpha(I) \alpha(J), \\
\alpha(I \cdot J) & =m(I) \alpha(J)+\alpha(I) m(J),
\end{aligned}
$$

so that the interval $K$ predicted by $(6.3)-(6.4)$ is too wide by the amount

$$
e(K, I \cdot J)=2 \alpha(I) \alpha(J)
$$

in this case.

Similarly, for relative precision, the formula

$$
a+b \cong \tilde{a}+\tilde{b} \text { r.p. } \ln \left(\frac{\tilde{a} e^{\alpha}+\tilde{b} e^{\beta}}{\tilde{a}+\tilde{b}}\right)
$$

is given in [4]. In terms of $g, \rho$ coordinates (Section 5), this defines the interval $K$ for which

$$
g(K)=g(I)+g(J), \quad \rho(K)=\frac{g(I) \rho(I)+g(J) \rho(J)}{g(I)+g(J)} .
$$

In standard format, one has

$$
I+J=\left[\frac{g(I)}{\rho(I)}+\frac{g(J)}{\rho(J)}, g(I) \rho(I)+g(J) \rho(J)\right],
$$

from which (5.17) and (6.8) give the excess width of $K$ to be

$$
e(K, I+J)=\frac{g(I) g(J)(\rho(I)-\rho(J))^{2}}{\rho(I) \rho(J)(g(I) \rho(I)+g(J) \rho(J))},
$$

which is positive if $\rho(I) \neq \rho(J)$. It is worth noting that $s(K)=s(I \cdot J)$ from (6.3)-(6.4), and $s(K)=s(I+J)$ in (6.8)-(6.9). Hence, the formulas of Olver give the correct upper limit of the interval containing the result.

Mathematics Research Center

University of Wisconsin-Madison

610 Walnut Street

Madison, Wisconsin 53706

1. G. H. Hardy, J. E. Littlewood \& G. Polya, Inequalities, 2nd ed., Cambridge Univ. Press, London, 1952.

2. R. E. Moore, Interval Analysis, Prentice-Hall, Englewood Cliffs, N. J., 1966.

3. R. E. MOORE, Methods and Applications of Interval Analysis, SIAM Studies in Applied Mathematics, Vol. 2, SIAM, Philadelphia, Pa., 1979.

4. F. W. J. Olver, "A new approach to error arithmetic," SIAM. J. Numer. Anal., v. 15, 1978, pp. 368-393.

5. H. RatscheK, “Representation of interval operations by coordinates," Computing, v. 24, 1980, pp. 93-96. 